\title{
Escala Pessoal de Crenças no Mundo Justo: Adaptação e Evidências de Validade
}

\author{
João Gabriel Modesto - Universidade de Brasilia, Brasília, Brasil \\ Universidade Estadual de Goiás, Goianésia, Brasil \\ Universidade de Brasilia, Brasilia, Brasil \\ Vanessa Figueredo - Universidade de Brasilia, Brasilia, Brasil \\ Guilherme Gama - Universidade de Brasilia, Brasília, Brasil \\ Matheus Rodrigues - Universidade de Brasília, Brasília, Brasil \\ Ronaldo Pilati - Universidade de Brasilia, Brasília, Brasil
}

\begin{abstract}
Resumo
As crenças no mundo justo (CMJ) têm permitido a compreensão de uma série de fenômenos sociais, o que tem levado ao desenvolvimento de medidas para sua mensuração. O presente artigo tem como objetivo apresentar evidências de validade de uma versão adaptada da Escala Pessoal de CMJ (CMJ-P). 146 participantes, em sua maioria mulheres (69\%), com idades variando de 17 a 66 anos $(M=27,79, D P=13,67)$ responderam ao instrumento de CMJ-P, a quatro escalas (CMJ-global, autoestima, bem-estar, religiosidade) para o teste de validade convergente e informaram dados sociodemográficos. Assim como no estudo original, foi encontrada uma solução unifatorial e consistência interna satisfatória $(\alpha=0,83)$. Adicionalmente, como esperado, foram encontrados relacionamentos positivos da CMJ-P com CMJ-G, autoestima e bem-estar, mas apenas marginalmente significativo com a religiosidade. Verificou-se ainda relação positiva da CMJ-P com a renda e nível de escolaridade. Esta versão adaptada demonstra-se adequada para uso em pesquisas.

Palavras-chaves: justiça social, vitimização, diferenças individuais, validade dos testes
\end{abstract}

\section{Personal Belief in a Just World Scale: Adaptation and Evidence of Validity}

\begin{abstract}
Belief in a just world (BJW) has allowed for the comprehension of a series of social phenomena, which has lead to the development of measures for its measurement. The present article has the aim to present evidence of validity for a Portuguese version of Personal BJW Scale (BJW-P). 146 participants, most of them women (69\%), with ages varying from 17 to 66 years $(\mathrm{M}=$ 27.79, DP = 13.67) answered PBJWP scale, four other scales (BJW-global, self-esteem, well-being, religiosity) for the convergent validity test and informed socio-demographic data. As in the original study, a uni-factorial solution and satisfactory internal consistency $(\alpha=0.83$ ) were found. Additionally, as expected, positive relations were found between PBJW and GBJW, self-esteem, and well-being, but only marginally significant with religiosity. Furthermore, positive relations of PBJW with income, as well as education, were found. The adapted version seems useful for research purposes.

Keywords: social justice, victimization, individual differences, validity of tests
\end{abstract}

\section{Escala Personal de Creencias en el Mundo Justo: Adaptación y Evidencias de Validez}

\section{Resumen}

Las creencias en un mundo justo (CMJ) han permitido la comprensión de una serie de fenómenos sociales, que han llevado al desarrollo de medidas para su medición. El presente artículo tiene como objetivo presentar evidencias de validez de una versión adaptada de la Escala Personal de CMJ (CMJ-P). Participaron 146 personas, en su mayoría mujeres (69\%), con edades entre 17 a 66 años $(M=27,79, \mathrm{DP}=13,67)$ que respondieron el instrumento de CMJ-P, a cuatro escalas (CMJ-global, autoestima, bienestar, religiosidad) para el test de validez convergente e informaron datos sociodemográficos. Así como en el estudio original, se encontró una solución unifactorial y consistencia interna satisfactoria $(\alpha=0,83)$. Además, se encontraron relaciones positivas de la CMJ-P con la CMJ-G, autoestima y bienestar, pero sólo marginalmente significativa con la religiosidad. Se encontró también, una relación positiva de la CMJ-P con los ingresos y el nivel de escolaridad. Esta versión adaptada es adecuada para su uso en investigaciones.

Palabras-clave: justicia social; persecusión; diferencias individuales; validez de los tests.

\section{Introdução}

Um time favorito ao título que foi desclassificado precocemente do campeonato, um político corrupto que é reeleito e famílias desabrigadas por conta de desastres naturais. O que essas situações, cuja natureza e complexidade dos fatos são tão diferentes entre si, podem ter em comum? Ao avaliar cada uma dessas situações, tende-se, muitas vezes, a fazer um julgamento sobre o grau de justiça e de merecimento dos envolvidos 
no fato. O time não jogou tão bem e mereceu perder? Cada povo tem o governante que merece? As pessoas, por destruírem o meio ambiente, são responsáveis pelos desastres naturais? Essas e muitas outras perguntas poderiam ser feitas no momento de avaliação das situações. Um conjunto amplo de pesquisas tem se interessado pelos processos psicológicos que permitem ao ser humano compreender a maneira como as pessoas fazem julgamentos relacionados à justiça (Lerner, 1980; Sidanius, Pratto, Laar, \& Levin, 2004; Tyler, 2006). É nesse conjunto de investigações que se situam os estudos sobre as crenças no mundo justo (CMJ).

De acordo com a hipótese do mundo justo (Lerner, 1980), as pessoas agem, ainda que de maneira não consciente (Hafer, 2000; Lerner, 1998), como se o mundo fosse um local controlável e ordenado (Correia \& Vala, 2003; Hafer \& Bègue, 2005), em que as pessoas têm o que merecem e merecem o que têm. Segundo a teoria, as pessoas não suportam viver em um mundo regido por eventos aleatórios, e as crenças no mundo justo surgem como um mecanismo psicológico adaptativo que permite uma sensação de invulnerabilidade pessoal e controle diante de um mundo instável (Hafer \& Rubel, 2015; Lerner, 1977).

Evidências do funcionamento da CMJ são descritos desde fases precoces do desenvolvimento infantil. Crianças tendem a avaliar mais positivamente outras crianças que são apresentadas como sortudas, em detrimento de crianças que são apresentadas como azaradas (Olson, Banaji, Dweck, \& Spelke, 2006). Segundo a hipótese do mundo justo, na situação avaliada, as crianças tendem a acreditar que a sorte não é algo tão imprevisível e que, de alguma forma, as crianças sortudas seriam merecedoras de sua sorte. Embora tenha sua origem na infância, a CMJ não se restringe a essa fase do desenvolvimento. Com o passar dos anos, diferentes fatores contribuiriam para a manutenção da CMJ (Hafer \& Rubel, 2015), a exemplo da meritocracia na cultura ocidental (Alves \& Correia, 2008) e de crenças religiosas.

Estudos têm indicado o potencial preditivo da $\mathrm{CMJ}$ em relação a uma série de variáveis. A sensação de controle e justiça oferecida pela CMJ tende a favorecer emoções positivas (Hafer \& Correy, 1999) e contribuir para o bem-estar subjetivo (Dalbert, 1999; Jiang, Yue, $\mathrm{Lu}, \mathrm{Yu}, \& \mathrm{Zhu}, 2015)$. No entanto, a manutenção da CMJ pode também assumir uma dimensão negativa. Em uma situação de vitimização, por exemplo, quando confrontado com uma vítima inocente, para manter a $\mathrm{CMJ}$, muitas vezes as pessoas acabam responsabilizando a vítima pela sua própria condição. Se existem vítimas inocentes, não é possível manter a crença de que o mundo é um lugar justo em que as pessoas têm o que merecem e merecem o que tem. Logo, para evitar a dissonância cognitiva, em muitos casos uma vítima inocente acaba sendo responsabilizada. $\mathrm{Na}$ medida em que a vítima deixa de ser avaliada como completamente inocente, é possível manter a crença de que o mundo é um lugar justo (Lerner \& Simmons, 1966).

Diferentes estudos têm corroborado as principais hipóteses relacionadas à $\mathrm{CMJ}$, e o desenvolvimento e a utilização de medidas de diferenças individuais que permitam mensurar o construto de maneira adequada exerce um importante papel no desenvolvimento de pesquisas na área. Nesse sentido, visando dar contribuições para o estudo da CMJ, o presente estudo teve como objetivo principal apresentar a adaptação da escala pessoal de CMJ para o português (Dalbert, 1999).

\section{Medidas de Diferenças Individuais da CMJ}

Quando Lerner formulou a hipótese do mundo justo, ele não focava no uso de medidas de diferenças individuais em suas pesquisas (Hafer \& Bègue, 2005), inclusive considera que algumas das escalas utilizadas para avaliar a CMJ são limitadas por serem alvo de forte desejabilidade social, uma vez que as sentenças das escalas costumam ser contranormativas. Uma alta concordância com os itens de algumas medidas indicaria um posicionamento irrealista e ingênuo de que as pessoas têm o que merecem (Lerner, 1998). Talvez, por conta disso, embora os primeiros estudos sobre a hipótese do mundo justo datem da década de 1960 (Lerner, 1965; Lerner \& Simmons, 1966), as primeiras escalas foram desenvolvidas apenas uma década depois (Rubin \& Peplau, 1975). Apesar das críticas e possíveis limitações de algumas medidas, o uso de escalas para avaliar a CMJ passou a ser usual na área (Furnham, 2003), sendo relevante o cuidado com seus parâmetros psicométricos.

A Escala do Mundo Justo (EMJ) (Rubin \& Peplau, 1975) é identificada na literatura como a primeira medida publicada para avaliar diferenças individuais da CMJ, embora uma versão dessa medida já tenha sido utilizada dois anos antes (Rubin \& Peplau, 1973). Além disso, outros instrumentos já buscavam avaliar crenças em um mundo justo \injusto como um fator de outros construtos (Collins, 1974).

Segundo Rubin e Peplau (1975), a EMJ, com 20 questões, deveria englobar itens para mensurar o alinhamento entre virtudes dos indivíduos e recompensas 
recebidas, avaliar a possibilidade de pessoas receberem recompensas sem terem merecido (ou de punições recebidas por pessoas com bons comportamentos). Os autores afirmam ainda o esforço na redação de assertivas considerando diferentes domínios da vida dos indivíduos: saúde, família, escola, política e justiça em relação a crimes.

Apesar da EMJ ter se tornado bastante popular em pesquisas internacionais que buscavam mensurar crenças relacionadas à justiça, o instrumento segue recebendo inúmeras críticas devido às propriedades psicométricas pouco desejáveis, baixa consistência interna e estrutura fatorial inconsistente (Gouveia, Pimentel, Coelho, Maynart, \& Mendonça, 2010; O'connor, Morrison, \& Morrison, 1996). Alguns estudos encontraram soluções unifatoriais (Ahmed \& Stewart, 1985), enquanto outros encontraram soluções multifatoriais (Hyland \& Dann, 1987; Whatley, 1993). Buscando uma alternativa para a EMJ, Lipkus (1991) desenvolveu a Escala Global de Crenças no Mundo Justo (EGCMJ), uma versão com número reduzido de itens, que vem apresentando melhores parâmetros psicométricos que a EMJ (O'connor et al., 1996). EGCMJ foi traduzida para o português, apresentando evidências favoráveis de validade (Gouveia et al., 2010).

\section{Crenças no Mundo Justo Pessoal e Global: Distinção entre os Tipos de CMJ}

Embora a EGCMJ sirva como opção ao uso da EMJ, ela se reduz ao julgamento que os indivíduos fazem sobre a justiça e merecimento em relação a outras pessoas. Apesar de a maioria dos estudos sobre a CMJ se referirem a essa dimensão, destaca-se a importância de investigações que analisem também as avaliações que os indivíduos fazem sobre justiça e merecimento para si próprios (Lerner \& Miller, 1978). É nesse sentido que se sustenta a distinção entre crenças no mundo justo pessoal (CMJ-P) e crenças no mundo justo global (CMJ-G). A primeira refere-se à avaliação de justiça e merecimento para si próprio, enquanto a segunda refere-se a uma avaliação feita para as pessoas em geral.

Lipkus, Dalbert e Siegler (1996) destacam a importância de considerar essas duas facetas da CMJ em separado, atentando que muitas vezes altos índices de uma dimensão de CMJ não implica em altos índices da outra dimensão (Dalbert, 1999). Baseado nas evidências disponíveis dos estudos sobre vieses atribucionais, Lipkus et al. (1996) propõem que os julgamentos que se faz de si mesmo e dos outros podem ser explicados por mecanismos distintos, que podem sofrer a interferência de vieses, como o viés de autosserviço (Miller \& Ross, 1975). Desse modo, para uma adequada compreensão sobre a CMJ, as dimensões pessoal e global deveriam ser avaliadas como construtos diferentes (ou complementares), que inclusive tendem a se relacionar e predizer fenômenos distintos (Alves \& Correia, 2013; Testé \& Perrin, 2013). A CMJ-P se relaciona positivamente com significados atribuídos à vida (Bègue \& Bastounis, 2003), com comportamentos altruístas (Bègue, Charmoillaux, Cochet, Cury, \& De Suremain, 2008), bem-estar subjetivo (Schaafsma, 2013), perdão (Strelan \& Sutton, 2011), autoestima (Dalbert, 1999). Já a CMJ-G se relaciona negativamente com comportamentos altruístas (Bègue et al., 2008) e positivamente com atitudes discriminatórias contra idosos, estigmatização de pobres, atitude favorável a penas punitivas (Bègue \& Bastounis, 2003), justificativas em relação às desigualdades sociais (Beierlein, Werner, Preiser, \& Wermuth, 2011), responsabilização e derrogação de vítimas (Correia, Alves, Morais, \& Ramos, 2015; Correia \& Vala, 2003; Modesto, 2014), bullying (Donat, Umlauft, Dalbert, \& Kamble, 2012).

Apesar da importância atribuída à distinção entre os tipos de CMJ, verifica-se que no Brasil há apenas instrumentos com evidências de validade para avaliar a CMJ-G (Gouveia et al., 2010). Feitas essas considerações, o presente estudo teve como objetivo apresentar evidências de validade de uma versão em português do instrumento desenvolvido por Dalbert (1999) para avaliar a dimensão pessoal da CMJ.

\section{Método}

\section{Participantes}

O Estudo contou com 146 participantes, sendo a maioria do sexo feminino $(69 \%)$ com idades variando de 17 a 66 anos $(M=27,79, D P=13,67)$. O grau de escolaridade variou de Ensino Fundamental completo $(0,7 \%)$ ao nível de Pós-graduação $(9,7 \%)$, com a maior parte da amostra concentrada em nível Superior Incompleto $(66,7 \%)$. A renda variou de um salário mínimo $(3,5 \%)$ até acima de 34 salários $(5,6 \%)$, com maior parcela da amostra relatando recebe entre oito e 13 salários (20,1\%), e entre 13 e $21(18,1 \%)$. Do total de participantes, $25,2 \%$ relataram serem ateus ou não possuírem nenhuma crença religiosa. Dos que afirmaram possuir alguma religião, houve uma maior parcela de católicos (36,4\%), seguidos por protestantes $(21,2 \%)$. O tamanho da amostra cumpre o critério de Pasquali (1999) de, ao menos, 10 participantes por item do instrumento. 


\section{Instrumentos}

CMJ Pessoal. Para o desenvolvimento da versão adaptada da medida, realizou-se um processo de tradução e retrotradução (Hambleton \& Zenisky, 2011). Uma equipe de pesquisadores fluente em inglês e português realizou a primeira tradução do instrumento original para o português. Em seguida, o instrumento traduzido foi encaminhado para outra equipe (também composta por pesquisadores fluentes nas duas línguas) que retrotraduziu o instrumento para o inglês. Realizou-se então uma comparação do instrumento original com a versão retrotraduzida. Após a realização de pequenos ajustes e novas comparações, foi alcançada uma versão final adequada do ponto de vista semântico. A escala pessoal de CMJ (Dalbert, 1999) é composta por sete itens associados a uma escala Likert variando de 1 (discordo totalmente) a 6 (concordo totalmente). A autora encontrou uma solução unifatorial para a CMJ pessoal, com valores satisfatórios de consistência interna $(\alpha=0,82)$. $\mathrm{O}$ instrumento de Dalbert (1999) é uma adaptação, realizada pela mesma autora, de versões anteriores da escala (Dalbert, Montada, \& Schmitt, 1987; Dalbert, 1993).

Buscando evidências de validade convergente, quatro instrumentos foram aplicados em conjunto com a versão traduzida da escala da CMJ-P. A escolha pelos instrumentos se deu em função de evidências da literatura de um relacionamento positivo entre as medidas e a CMJ-P (Furnham, 2003), bem como de comparações utilizadas no estudo de validação da medida original (Dalbert, 1999).

CMJ Global. Foi utilizada a versão traduzida para o português (Gouveia et al., 2010) da Escala Global de Crenças no Mundo Justo (Lipkus, Dalbert, \& Siegler, 1996), que, no estudo de adaptação, apresentou um alfa de Cronbach de 0,71. A escala obteve um índice de confiabilidade adequado na amostra investigada no presente estudo ( $\alpha=0,88)$.

Bem-Estar Subjetivo. Foi também utilizada a subescala de Satisfação com a Vida da Medida de Bem-Estar Subjetivo (Albuquerque \& Tróccoli, 2004). A medida apresentou consistência interna satisfatória no estudo de validação $(\alpha=0,90)$ e no presente estudo $(\alpha=0,91)$.

Autoestima. Uma versão adaptada para o português da escala de autoestima de Rosenberg (Dini, Quaresma, \& Ferreira, 2004) foi aplicada, sendo encontrados índices satisfatórios de confiabilidade no presente estudo ( $\alpha=0,87)$.

Religiosidade. Uma versão traduzida (Taunay et al., 2012) do índice de religiosidade de Duke (DUREL)
(Koening \& Büssing, 2010) foi utilizada para avaliar a religiosidade. $\mathrm{O}$ instrumento avalia crenças religiosas e a frequência de comportamentos relacionados à religião. A medida apresentou índices satisfatórios de confiabilidade no presente estudo $(\alpha=0,86)$.

\section{Procedimento de Coleta e Análise de Dados}

A coleta foi realizada a partir de questionários em formato lápis e papel. Os questionários foram aplicados individualmente na Universidade de Brasília em estudantes universitários e também em transeuntes em locais públicos de Brasília. A ordem em que as escalas foram apresentadas foi: CMJ pessoal, CMJ global, bem-estar subjetivo, autoestima, religiosidade e dados sociodemográficos.

A estrutura da CMJ pessoal foi analisada por meio de uma análise fatorial exploratória com método de extração PAF (Principal Axis Factoring). Utilizou-se ainda o critério do autovalor (eigenvalue) para extração dos fatores. Para o teste de fidedignidade, foi conduzida uma análise de consistência interna com o alfa de Cronbach. Foram ainda realizados testes de correlação de Pearson, a fim de testar a validade convergente da CMJ pessoal com os demais instrumentos aplicados. Por último, foram realizadas análises de variância (ANOVA) e novamente testes de correlação para averiguar a relação da $\mathrm{CMJ}$ pessoal com variáveis sociodemográficas.

\section{Resultados}

\section{Análise Fatorial Exploratória}

Antes de proceder à análise fatorial exploratória (AFE), buscou-se verificar se a matriz de correlação era fatorável. O índice de $\mathrm{KMO}(0,85)$ e o Teste de Esfericidade de Bartlett, $\chi^{2}(21)=380,42, p<0,001$, indicaram fatorabilidade da matriz, permitindo a realização da AFE. A partir do critério de autovalor (eigenvalue) superior a um, verificou-se a existência de um único fator com 45,84\% de variância explicada. A estrutura fatorial encontrada está de acordo com o relatado na versão original do instrumento (Dalbert, 1999).

A análise das cargas fatoriais aponta que todos os itens apresentaram carga fatorial acima de 0,30, variando de 0,47 a 0,85 , indicando que todos parecem adequados para a composição do fator. As cargas fatoriais de cada item e as comunalidades estão dispostas na Tabela 1. Como também pode ser observada na Tabela 1, a consistência interna demonstrou-se satisfatória $(\alpha=0,83)$. 
Tabela 1

Cargas Fatoriais, Comunalidades eConsistência Interna da Escala de CMJ-P

\begin{tabular}{lcc}
\hline Item & $\begin{array}{c}\text { Carga } \\
\text { fatorial }\end{array}$ & $\boldsymbol{b}^{\mathbf{2}}$ \\
\hline 04. De modo geral, os acontecimentos da minha vida são justos. & 0,85 & 0,72 \\
06. Eu acredito que a maioria das coisas que acontecem comigo são justas. & 0,82 & 0,67 \\
03. Eu acredito que geralmente tenho o que mereço. & 0,70 & 0,48 \\
02. Eu geralmente sou tratado de forma justa. & 0,66 & 0,43 \\
01. Eu acredito que, geralmente, eu mereço o que acontece comigo. & 0,61 & 0,37 \\
07. Eu acredito que importantes decisões tomadas ao meu respeito geralmente são justas. & 0,56 & 0,32 \\
05. Na minha vida a injustiça é exceção mais do que a regra. & 0,47 & 0,22 \\
\hline Variância explicada & $\mathbf{4 5 , 8 2 \%}$ \\
\hline Alfa de Cronbach & $\mathbf{0 , 8 3}$ & \\
\hline
\end{tabular}

\section{Evidências de Validade Convergente}

Após a condução da AFE e avaliação da consistência interna da CMJ pessoal, foi dado seguimento ao teste de validade convergente. A partir de testes de correlação de Pearson, verificou-se que, como hipotetizado, a CMJ pessoal se relacionou positivamente com a CMJ global $(r=0,57, p<0,001)$, com o bem-estar $(r=0,45, p<0,001)$ e com a autoestima $(r=0,38, p<$ $0,001)$. No entanto, diferente do esperado, não houve relação com a religiosidade $(r=0,01, p=0,49)$. Considerando que os estudos que investigaram a relação de religiosidade com a CMJ utilizaram amostras com participantes de religiões cristãs, e a amostra do presente estudo englobou participantes de religiões de diferentes preceitos e não religiosos decidiu-se por fazer uma análise apenas com participantes que se declararam cristãos (i.e., católicos e prostestantes, $n=83$ ). Quando avaliados apenas esse grupo, a relação entre $\mathrm{CMJ}$ pessoal e religiosidade tornou-se marginalmente significativa, $r=0,18 ; p=0,053$.

Buscando conhecer mais sobre a CMJ pessoal, a relação entre o construto e as variáveis sociodemográficas consideradas no presente estudo foi testada. A partir de uma ANOVA, não foram encontradas diferenças de sexo, $F(1,143)=0,71, p=0,372, \eta^{2} p=0,006$. Em relação à idade, a partir de um teste de correlação de Pearson, também não foram encontradas relações significativas, $r=0,041, p=0,630$. Já em relação à escolaridade, verificou-se que quanto maior o índice de escolaridade maiores índices de CMJ pessoal, $r=0,28$, $p=0,001$. Também foi encontrado um relacionamento positivo com a renda dos participantes $(r=0,17, p=$
0,019), indicando que maiores índices de CMJ pessoal estão relacionados a uma maior renda dos participantes.

\section{Discussão}

O presente estudo teve como objetivo apresentar evidências de validade da versão traduzida da escala de Dalbert (1999) para avaliar a dimensão pessoal da CMJ. Foi avaliada a estrutura fatorial da escala, a consistência interna e testada a validade convergente do instrumento com medidas de bem-estar, autoestima e religiosidade.

Assim como no estudo de validação da medida original, foi encontrada uma solução unifatorial. Considerando que há um debate na área sobre estrutura fatorial da CMJ (Hyland \& Dann, 1987; Whatley, 1993), os achados do presente estudo tendem a corroborar a hipótese de uma solução unifatorial, ao menos se considerada exclusivamente a dimensão pessoal da CMJ. É possível que a multidimensionalidade encontrada em outras escalas seja explicada pela falta de clareza na separação das dimensões pessoal e global. Quando considerados itens bem definidos para ambas as dimensões, as escalas apresentam soluções bifatoriais, sendo um fator para a dimensão global e outro fator para a dimensão pessoal (Dalbert, 1999).

Em relação à precisão da medida, foram encontrados valores de alfa de Cronbach satisfatórios $(\alpha=$ $0,83)$ e bem próximo aos valores encontrados em Dalbert (1999) $(\alpha=0,82)$. O índice de confiabilidade encontrado corrobora outros estudos que indicam que as medidas mais breves da $\mathrm{CMJ}$, com menor número de itens, parecem mais adequadas que medidas mais 
extensas, ao menos em termos de confiabilidade da medida (O'connor et al., 1996).

O teste de validade convergente também corroborou os achados da medida original, sendo encontrados relacionamentos positivos da CMJ-P com a CMJ-G, autoestima e com o bem-estar. Como postulado pela hipótese do mundo justo, uma vez que o indivíduo acredita que ele próprio "tem o que merece e merece o que tem", (Lerner, 1980) isso contribui positivamente com o seu bem-estar e autoestima. A ideia básica é que avaliar-se em uma situação de injustiça gera reações negativas. Logo, maiores índices de CMJ pessoal, por representarem a crença de que há um senso de justiça em relação a si mesmo, tendem a se associar com construtos como bem-estar subjetivo e autoestima.

A relação da CMJ pessoal com a religiosidade, diferente do que era esperado, foi apenas marginalmente significativa e somente quando considerados os participantes de religiões cristãs. Destaca-se que outros estudos também encontraram relações apenas modestas da CMJ com a religiosidade (Crozier \& Joseph, 1997), a despeito de evidências de relações mais fortes entre os construtos (Kurst, Bjorck, \& Tan, 2000). É preciso então avaliar quais moderadores e mediadores interferem nessa relação. É possível que fatores culturais exerçam algum papel nessa relação. No caso de estudos realizados no Brasil, por exemplo, há outras evidências de ausência de relação da CMJ (dimensão global) com a religiosidade (Gouveia et al., 2010), diferente do que predomina em estudos realizados na Europa e Estados Unidos.

Sobre o relacionamento da CMJ pessoal com variáveis sociodemográficas, não foram encontradas diferenças de idade e sexo (Durm \& Stowers, 1998), o que favorece a utilização dessa medida em grupos de diferentes faixas etárias e de ambos os sexos. Esse achado, no entanto, deve ser considerado de maneira parcimoniosa, já que a maioria da amostra foi composta por estudantes universitários e, em sua maioria, mulheres. Já em relação à renda e nível de escolaridade, foram encontradas relações positivas com a CMJ pessoal. Uma vez que renda e nível de escolaridade estão associados a maior status e poder, é possível que esse resultado seja explicado pelo papel exercido pela CMJ enquanto mecanismo justificador do sistema na manutenção do status quo (Jost \& Banaji, 1994; Lima-Nunes, Pereira, \& Correia, 2013). Pessoas com mais status e poder tendem a aceitar que o mundo é um local justo para si mesmo, e a concluir que sua posição se deve ao esforço empreendido.
Apesar das evidências de validade da medida e, consequentemente, de preencher uma lacuna na literatura nacional (Modesto, 2014), o presente estudo possui algumas limitações. A amostra utilizada foi restrita ao Distrito Federal e, em sua grande maioria, composta por estudantes universitários. Buscando um incremento da validade ecológica, seria adequado que novos estudos testassem a medida em contextos mais diversos, variando o perfil da amostra. Além disso, para avaliação da estrutura fatorial da medida, limitou-se à condução de uma análise fatorial exploratória. Novos estudos podem lançar mão de uma estratégia de análise fatorial confirmatória, tomando como base os achados encontrados na presente pesquisa.

Considerando as evidências de validade favoráveis, a escala traduzida no presente estudo poderá ser utilizada para estudos sobre a CMJ no contexto brasileiro. $\mathrm{O}$ uso de medidas traduzidas e adaptadas favorece comparações transculturais, permitindo assim avaliar a extensão cultural das predições sobre a CMJ, um campo de investigação que tem sido de interesse de pesquisadores na avaliação das diferenças individuais da CMJ (Furnham, 2003).

Além dos parâmetros psicométricos favoráveis, a escala pessoal da CMJ ainda possui a vantagem de ser uma medida breve e de fácil aplicação, podendo ser utilizada, sem maior ônus de tempo, em delineamentos experimentais ou em estudos correlacionais com outros instrumentos.

\section{Referências}

Ahmed, S. M. S., \& Stewart, R. A. C. (1985). Factor analytical and correlational study of just world scale. Perceptual and Motor Skills, 60, 135-140. doi: 10.2466/pms.1985.60.1.135

Albuquerque, A. S., \& Tróccoli, B. (2004). Desenvolvimento de uma escala de bem-estar subjetivo. Psicologia: Teoria e Pesquisa, 20(2), 153-164.

Alves, H., \& Correia, I. (2008). On the normativity of expressing the Belief in a Just World: Empirical evidence. Social Justice Research, 21(1), 106-118. doi:10.1007/s11211-007-0060-x

Alves, H., \& Correia, I. (2013). The bufferingboosting hypothesis of the expression of general and personal belief in a just world for succeses and failures. Social Psychology, 44, 390-397. doi:10.1027/1864-9335/a000151 
Bègue, L., \& Bastounis, M. (2003). Two spheres of belief in justice: Extensive support for the bidimensional model of belief in a just world. Journal of Personality, 71(3), 435-63. doi: 10.1111/1467-6494.7103007

Bègue, L., Charmoillaux, M., Cochet, J., Cury, C., \& De Suremain, F. (2008). Altruistic behavior and the bidimensional just world belief. The American Journal of Psychology, 121(1), 47-56. doi: 10.2307/20445443

Beierlein, C., Werner, C. S., Preiser, S., \& Wermuth, S. (2011). Are just-world beliefs compatible with justifying inequality? Collective political efficacy as a moderator. Social Justice Research, 24(3), 278-296. doi:10.1007/s11211-011-0139-2

Collins, B. E. (1974). Four components of the rotter internal - external scale: Belief in a difficult world, a just world, a predictable world, and a politically responsive world. Journal of Personality and Social Psychology, 29(3), 381-391. doi: 10.1037/h0036015

Correia, I., Alves, H., Morais, R., \& Ramos, M. (2015). The legitimation of wife abuse among women: The impact of belief in a just world and gender identification. Personality and Individual Differences, 76, 7-12. doi:10.1016/j.paid.2014.11.041

Correia, I., \& Vala, J. (2003). Crença no mundo justo e vitimização secundária : O papel moderador da inocência da vítima e da persistência do sofrimento. Análise Psicológica, 3(XXI), 341-352.

Crozier, S., \& Joseph, S. (1997). Religiosity and sphere-specific just world beliefs in 16 to 18 year olds. Journal of Social Psychology, 137, 510-513. doi: 10.1080/00224549709595468

Dalbert, C. (1993). The personal belief in a just world scale (PBJWS). Unpublished scale.

Dalbert, C. (1999). The world is more just for me than generally : About the personal belief in a just world scale's validity. Social Justice Research, 12(2), 79-98. doi:10.1023/A:1022091609047

Dalbert, C., Montada, L., \& Schmitt, M. (1987). Glaube an eine gerechte welt als motiv: Validierungskorrelate zweier Skalen (Belief in a just world: Validity correlates of two scales). Psychologische Beitrage 29, 596-615.

Dini, G. M., Quaresma, M. R., \& Ferreira, L. M. (2004). Adaptação cultural e validação da versão brasileira da escala de autoestima de Rosenberg. Revista da Sociedade Brasileira de Cirurgia Plástica, 19(1), 41-52.
Donat, M., Umlauft, S., Dalbert, C., \& Kamble, S. V. (2012). Belief in a just world, teacher justice, and bullying behavior. Aggressive Behavior, 38(3), 185193. doi:10.1002/ab.21421

Durm, M., \& Stowers, D. (1998). Just world beliefs and irrational beliefs: A sex difference? Psychological Reports, 83, 328-330. doi: 10.2466/pr0.1998.83.1.328

Furnham, A. (2003). Belief in a just world: Research progress over the past decade. Personality and Individual Differences, 34(5), 795-817. doi:10.1016/ S0191-8869(02)00072-7

Gouveia, V. V., Pimentel, C. E., Coelho, J. A. P. de M., Maynart, V. A. P., \& Mendonça, T. dos S. (2010). Validade fatorial confirmatória e consistência interna da Escala Global de Crenças no Mundo Justo - GJWS. Interação Em Psicologia, 14(1), 21-29.

Hafer, C. L. (2000). Do innocent victims threaten the belief in a just world? Evidence from a modified Stroop Task. Journal of Personality and Social Psychology, 79(2), 165-173. doi:10.1037/AW22-3514.79.2.165

Hafer, C. L., \& Bègue, L. (2005). Experimental research on just-world theory: Problems, developments, and future challenges. Psychological Bulletin, 131(1), 128-67. doi:10.1037/0033-2909.131.1.128

Hafer, C. L., \& Correy, B. L. (1999). Mediators of the relation between beliefs in a just world and emotional responses to negative outcomes. Social Justice Research, 12 (3), 189-204. doi: 10.1023/A:1022144317302

Hafer, C. L., \& Rubel, A. N. (2015). The why and how of defending belief in a just world. Em James M. Olson and Mark P. Zanna (Eds.), Advances in Experimental Social Psychology (pp.41-96). doi:10.1016/bs.aesp.2014.09.001

Hambleton, R. K., \& Zenisky, A. L. (2011). Translating and adapting tests for cross-cultural assessments. Em D. Matsumoto \& F. J. R. van de Vijver (Eds.), Cross-Cultural Research Methods in Psychology (pp. 4670). New York: Cambridge.

Hyland, M. E., \& Dann, P. L. (1987). Exploratory factor analysis of the Just World Scale using british undergraduates. British Journal of Social Psychology, 26, 73-77. doi: 10.1111/j.2044-8309.1987.tb00762.x

Jiang, F., Yue, X., Lu, S., Yu, G., \& Zhu, F. (2015). How Belief in a Just World benefits mental health: The 
effects of optimism and gratitude. Social Indicators Research, 1-13. doi:10.1007/s11205-015-0877-x

Jost, J. T., \& Banaji, M. R. (1994). The role of stereotyping in system-justification and the production of false consciousness. British Journal of Social Psycho$\log y$, 33(1), 1-27. doi:10.1111/j.2044-8309.1994. tb01008.x

Koenig, H. G., \& Büssing, A. (2010). The Duke University Religion Index (DUREL): a five-item measure for use in epidemological studies. Religions, 1, 7885. doi: 10.3390/rel1010078

Lerner, M. J. (1965). Evaluation of performance as a function of performer's reward and attractiveness. Journal of Personality and Social Psychology, 1(4), 355360. doi:10.1037/h0021806

Lerner, M. J. (1977). The justice motive: Some hypotheses as to its origins and forms. Journal of Personality, 45(1), 1-52. doi:10.1111/j.1467-6494.1977. tb00591.x

Lerner, M. J. (1980). The belief in a just world: A Fundamental delusion (p. 209). New York: Plennum Press.

Lerner, M. J. (1998). The two forms of belief in a just world: Some thoughts on why and how people care about justice. Em L. Montada \& M. J. Lerner (Eds.), Responses to victimizations and belief in a just world (pp. 247-270). New York: Plenum Press.

Lerner, M. J., \& Miller, D. T. (1978). Just world research and the attribution process: Looking back and ahead. Psychological Bulletin, 85(5), 1030-1051. doi:10.1037//0033-2909.85.5.1030

Lerner, M. J., \& Simmons, C. H. (1966). The observer's reaction to the "innocent victim": Compassion or rejection? Journal of Personality and Social Psychology, 4(2), 203-210. doi:10.1037/h0023562

Lima-Nunes, A., Pereira, C. R., \& Correia, I. (2013). Restricting the scope of justice to justify discrimination: The role played by justice perceptions in discrimination against immigrants. European Journal of Social Psychology, 43, 627-636. doi:10.1002/ ejsp.1981

Lipkus, I. (1991). The construction and preliminary validation of a global belief in a just world scale and the exploratory analysis of the multidimensional belief in a just world scale. Personality and Individual Differences, 12, 1171-1178. doi: 10.1016/0191-8869(91)90081-L
Lipkus, I. M., Dalbert, C., \& Siegler, I. C. (1996). The importance of distinguishing the Belief in a Just World for self versus for others: Implications for psychological well-being. Personality and Social Psychology Bulletin, 22(7), 666-677. doi:10.1177/0146167296227002

Kurst, J., Bjorck, J., \& Tan, S. (2000). Causal attributions for uncontrollable negative events. Journal of Psychology and Christianity, 19, 47-60.

Miller, D. T., \& Ross, M. (1975). Self-serving biases in the attribution of causality: Fact ou fiction? Psychological Bulletin, 82, 213-225. doi: 10.1037/h0076486

Modesto, J. G. N. (2014). “Nem todas as vítimas importam”: A influência das crenças no mundo justo na responsabilização de vítimas de diferentes grupos sociais (Dissertação de mestrado). Universidade de Brasília, Brasília-DF.

O'connor, W. E., Morrison, T. G., \& Morrison, M. A. (1996). The reliability and factor structure of the global belief in a just world scale. The Journal of Social Psychology, 136(5), 667-668. doi:10.1080/0022 4545.1996.9714055

Olson, K. R., Banaji, M. R., Dweck, C. S., \& Spelke, E. S. (2006). Children's biased evaluations of lucky versus unlucky people and their social groups. Psychological Science, 17(10), 845-846. doi: 10.1111/j.1467-9280.2006.01792.x

Pasquali, L (1999). Instrumentos psicológicos: Manual prático de elaboração. Brasilia: LabPAM.

Rubin, Z., \& Peplau, L. A (1973). Belief in a just world and reactions to another's lot: A study of participants in the national draft lottery. Journal of Social Issues, 29(4), 73-93.

Rubin, Z., \& Peplau, L. A. (1975). Who believes in a just world? Journal of Social Issues, 31(3), 65-89. doi:10.1111/j.1540-4560.1975.tb00997.x

Schaafsma, J. (2013). Through the lens of justice: Just world beliefs mediate relationships between perceived discrimination and subjective well-being. International Journal of Intercultural Relations, 37(4), 450-458. doi:10.1016/j.ijintrel.2013.04.002

Sidanius, J., Pratto, F., Laar, C. Van, \& Levin, S. (2004). Social Dominance Theory: its agenda and method. Political Psychology, 25(6), 845-880.

Strelan, P., \& Sutton, R. M. (2011). When just-world beliefs promote and when they inhibit forgiveness.

Psico-USF, Bragança Paulista, v. 22, n. 1, p. 13-22, jan./abr. 2017 
Personality and Individual Differences, 50(2), 163-168. doi:10.1016/j.paid.2010.09.019

Taunay, T. C. D., Gondim, F. D. A. A., Macêdo, D. S., Moreira-Almeida, A., Gurgel, L. D. A., Andrade, L. M. S., \& Carvalho, A. F. (2012). Validação da versão brasileira da escala de religiosidade de Duke (DUREL). Revista de Psiquiatria Clinica, 39(4), 130135. doi:10.1590/S0101-60832012000400003

Testé, B., \& Perrin, S. (2013). The impact of endorsing the belief in a just world on social judgments: The social utility and social desirability of Just-World Beliefs for self and others. Social Psychology, 44(3), 209-218. doi:10.1027/1864-9335/a000105
Tyler, T. R. (2006). Psychological perspectives on legitimacy and legitimation. Annual Review of Psychology, 57, 375-400. doi:10.1146/annurev. psych.57.102904.190038

Whatley, M. A. (1993). Belief in a Just World Scale: unidimensional or multidimensional? The Journal of Social Psychology, 133(4), 547-551. doi:10.1080/002 24545.1993.9712180

Recebido em: 19/02/2015

Reformulado em: 30/11/2015

Aceito em: 15/12/2015 
Nota dos autores:

O presente trabalho recebeu apoio do CNPq na forma de Bolsa Produtividade em Pesquisa (PQ) e apoio individual no Edital Universal CNPq (478285/2013-2) para o quinto autor.

Sobre os autores:

João Gabriel Modesto é Professor Assistente da Universidade Estadual de Goiás e doutorando no Programa de Pós-Graduação em Psicologia Social, do Trabalho e das Organizações (PSTO) da Universidade de Brasília. É também membro do Laboratório de Psicologia Social (LAPSOCIAL-UnB).

E-mail: jg.modesto@gmail.com

Vanessa Figueredo é graduanda em psicologia pela Universidade de Brasília e membro do Laboratório de Psicologia Social (LAPSOCIAL-UnB).

Guilherme Gama é graduando em psicologia pela Universidade de Brasília e membro do Laboratório de Psicologia Social (LAPSOCIAL-UnB).

Matheus Rodrigues é graduando em psicologia pela Universidade de Brasília e membro do Laboratório de Psicologia Social (LAPSOCIAL-UnB).

Ronaldo Pilati possui Doutorado em Psicologia pela Universidade de Brasília. É professor associado do Departamento de Psicologia Social e do Trabalho (PST) e do Programa de Pós-Graduação em Psicologia Social, do Trabalho e das Organizações (PSTO) da UnB. É também coordenador do Laboratório de Psicologia Social (LAPSOCIAL-UnB). E-mail: rpilati@gmail.com

\section{Contato com os autores:}

João Gabriel Modesto

Universidade de Brasília, Campus Universitário Darcy Ribeiro, Instituto Central de Ciências, Instituto de Psicologia Brasília-DF, Brasil

CEP: 70910-900 